\title{
The University of Wisconsin-Madison Torsatron/Stellarator Laboratory Program
}

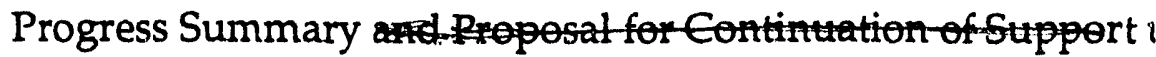 \\ DE-FG02-86ER53216.A010 \\ October, 1992
}

DOE/ER/53216 - -13

A summary of results obtained during the past year of the current grant for research at the University of Wisconsin-Madison Torsatron/Stellarator Laboratory is presented along with goals for the next funding period. Research efforts have focussed on fundamental physics issues associated with toroidal confinement, predominantly through experimental investigations on the Interchangeable Module Stellarator (IMS), in the topics of fluctuations, potentials and electric fields, and plasma rotation and damping. Theoretical and computational activities have also been undertaken to support the experimental research and to identify interesting new toroidal confinement concepts which could contribute to the understanding of tokamak transport.

Accomplishments during this grant period include:

- Measurement of the plasma potential, plasma density, and their fluctuations over an entire poloidal cross-section have been made.

- Calculation of the Reynolds stress driven poloidal flow compares well with the flow computed from plasma equilibrium profiles.

- Electrode biasing in the edge regions to generate a $3-4 \mathrm{kV} / \mathrm{m}$ positive radial electric field which drives a poloidal rotation of $10-15 \mathrm{~km} / \mathrm{s}$.

- Two dimensional measurements showing there are significant asymmetries in the fluctuation-induced transport on a magnetic surface.

- For non-biased conditions, the particle flux is predominantly on the inboard and outboard sides of the torus. In the biased case, observation of a significant reduction of outward transport around the entire surface accompanied by an inward motion of the density profile peaks.

- Development of a model based on the fluid equations to describe plasma rotation, ambipolar electric fields, and radial electrical conductivity in a nonsymmetric toroidal plasma subject to an external biasing electrode.

- Direct measurement of poloidal flow profiles which are in good agreement with force balance calculations under various operating conditions.

- Preliminary measurements of the poloidal rotation decay rates and radial conductivity showing the proper scaling with neutral pressure in agreement with the neoclassical fluid model.

- Development of a code to calculate the Boozer coordinates from actual conductors for application to transport codes and data analysis.

- Efforts underway to determine whether conditions in IMS can exist which increase the contribution of viscosity to rotation damping and condur "vity.

- Investigation of quasi-helically symmetric systems to assess their potential role in contributing to the understanding of tokamak confinement. The HSX proposal was an outgrowth of these investigations. 


\section{Fluctuation-Induced Transport Experiments}

Current studies on the Interchangeable Module Stellarator (IMS) in the area of fluctuation-induced transport are designed to address key issues in determining the role of this plasma loss mechanism in toroidal confinement devices. In IMS, the Reynolds-stress-driven poloidal flows compare well with flows computed from plasma equilibrium profiles; this mechanism is a proposed drive for the poloidal rotation seen in the L-H transition[1]. Induced radial electric fields and poloidal flows from the electrode-biasing experiments cause the fluctuation-induced transport to be significantly reduced.

Measurements of the plasma potential, plasma density, and fluctuations in these quantities, over an entire poloidal cross section of ECH plasma ( $B=0.26 \mathrm{~T}, \mathrm{P}=$ $1 \mathrm{~kW},\left\langle\mathrm{n}_{\mathrm{e}}\right\rangle=5-11 \times 10^{10} \mathrm{~cm}^{-3}, \mathrm{~T}_{\mathrm{e}}=5-10 \mathrm{eV}, \mathrm{T}_{\mathrm{i}}=1-4 \mathrm{eV}$ ) have been made using an array of emissive and Langmuir probes. The spatial orientation and spacing of the four emissive probes provides a measurement of the plasma potential and its 3-dimensional gradient over a small cell of the plasma volume. From these measurements, one can compute the Reynolds stress $\left(\left\langle\mathrm{v}_{\mathbf{r}} \mathrm{v}_{\theta}\right\rangle\right)$, and along with the corresponding local density fluctuations, the electrostatic fluctuation-induced transport $\left(\left\langle\mathrm{n}_{\mathrm{e}} \mathrm{v}_{\mathrm{r}}\right\rangle\right)$.

These parameters have been measured for two distinct conditions created in IMS discharges of 10-ms duration: (1) An electrode placed $1 \mathrm{~cm}$ inside the separatrix is biased to $+80 \mathrm{~V}$ which creates a strong positive radial electric field $(3-4 \mathrm{kV} / \mathrm{m})$ and drives a large poloidal rotation of the edge plasma $(10-15 \mathrm{~km} / \mathrm{s})$; (2) The electrode is electrically disconnected from its source, and the plasma is allowed to relax to its nominal state.

From the measured radial electric field and pressure profiles, IMS exhibits a net poloidal rotation in the unbiased case, where there is no external driving force. It has been suggested that fluctuations are coupled to poloidal flow through the Reynolds stress tensor [1]. The Reynolds-stress-driven poloidal flow is estimated by:

$$
v_{\theta}=\gamma\left\{\frac{\partial<v_{r} v_{\theta}>}{\partial r}\right\}
$$

where $\gamma$ is the poloidal damping time, the braces $\{. .$.$\} indicates an average over$ the magnetic flux surface, and the angle brackets <...> indicates an ensemble average of the velocity components $v_{r}$ and $v_{\theta}$. A poloidal damping time of 19-24 $\mu s$ is determined from the measured time response of poloidal plasma flow to pulsed electrode biasing. The estimated Reynolds stress induced flow of $600 \pm 350 \mathrm{~m} / \mathrm{s}$ in the IMS edge region agrees well with the value of $500 \pm 150 \mathrm{~m} / \mathrm{s}$ determined by force balance, as is shown in Fig. 1. 
The two-dimensional measurements show there are significant asymmetries in the fluctuation-induced transport on a magnetic flux surface. Figure 2 shows the radially-directed flux density decomposed onto magnetic flux surfaces for the biased and unbiased conditions at three minor radii. At the separatrix (top graph), the flux is directed mainly on the outside $\left(\theta=0^{\circ}\right)$ and inside regions of the torus for the unbiased plasma while the biased case shows significant reduction in transport for the entire surface. The transport at $\mathrm{r} / \mathrm{a}=0.7$ shows inward transport on the top of the device $\left(\theta=90^{\circ}\right)$ for both conditions. The bottom graph is indicative of the transport for $\mathrm{r} / \mathrm{a}<0.5$.

Comparisons with measurements of particle confinement times show that the fluctuation-induced transport is a major particle loss mechanism in the nominal (no electrode bias) discharges. Estimates of this loss rate when compared to the total particle loss rate, measured from the sum of divertor particle fluxes, represents $46 \%$ of the total particle losses in nominal IMS discharges. However, the significant reduction of the fluctuation-induced transport during electrode biasing does not result in enhanced confinement in IMS since the current required to maintain the electrode voltage represents a significant loss channel for the electrons. The magnitude of the electrode current is $\approx 50 \%$ of the equivalent current of the total divertor particle flux and, is thus comparable to the fluctuation-induced particle flux of the unbiased plasma.

Fluctuation studies during the upcoming grant period will be a combination of additional experiments to corroborate the present data base and investigate the effects of heating location, and a more detailed analysis of the existing data base to examine relevance of various proposed poloidal flow generation mechanisms. Additional data runs will be comprised of another 2-D scan of the fluctuation levels and transport at a lower magnetic field strength ( $2.4 \mathrm{kG}$ on axis) where the ECH resonance region is predominantly on the inboard side of the device and the particle confinement time has been shown to be significantly greater than the case of $2.6 \mathrm{kG}$ on axis. Ion temperature measurements will be made using a gridded-energy analyzer to determine the de'ailed profiles necessary to compute the equilibrium poloidal velocity with suf'inient accuracy to compare with the flows calculated for the Reynolds stress and other possible flow generation mechanisms. Specifically, data will be interpreted to compute the driving term and applicability of the "Stringer Spin-up" poloidal flow generation mechanism ( asymmetric radial fluxes coupled to poloidal momentum) for comparison with the equilibrium and Reynolds stress flows.

The emissive/Langmuir probe array permits the calculation of the three dimensional wavenumber spectra of the fluctuations which are used to compare with predicted dispersion relationships for the leading possible mechanisms for driving the fluctuations (drift instabilities, resistive interchange modes, etc. ). Data analysis will continue to provide insight into the structure and possible causes of the fluctuations observed. 
2. Evolution of the Plasma Rotation and Radial Electric Field in Toroidal Plasmas Subject to a Biased Electrode

With the observation of the $\mathrm{L}-\mathrm{H}$ transition and the experimentally observed changes in plasma rotation and the radial electric field, the problem of determining the ambipolar electric field and plasma rotation in a tokamak has received renewed interest $[2,3]$. To artificially induce the $\mathrm{L}-\mathrm{H}$ transition in tokamaks, strong radial electric fields have been set up through the application of an electrode bias[4,5]. Biased-electrode-induced plasma rotation has been observed to reduce fluctuation induced transport in IMS as well, as just reported. The radial current (set up in these cases through the biased electrode technique) can just as well be produced by ions in loss orbits as proposed in the model by Shaing[2], non-ambipolar electron losses as described by Itoh [6], or anomalous viscosity as assumed by Rozhanskii and Tendler[7].

A model framework has been developed to facilitate experimental investigations of plasma rotation, radial electric conductivities and electric fields in IMS with a biased electrode. The fluid equation approach has been used to analyze the time evolution of the plasma rotation and the ambipolar electric field in a nonsymmetric toroidal plasma subject to an external biasing voltage induced by a probe. The model considers a plasma in the Pfirsch-Schlüter or the plateau regime and includes the effects of a background neutral gas. A time-dependent charge conservation equation is used to determine the ambipolar electric field as a function of time. It is found that after the application of the biasing voltage, the electric field and plasma rotation change quickly and reach steady state after a time inversely proportional to the sum of the momentum damping rates due to viscosity and ion-neutral collisions. The steady-state is characterized by a radial electric field and a plasma rotation that are proportional to the electric current flowing through the biasing probe. The direction of the plasma flow is determined by the relative magnitude of the momentum damping rates on the flux surface. firom the steady-state solution, an expression for the radial electric conductivity is obtained, which includes the effect of collisions with neutrals as well as viscosity. The full details and equations of the model are available in the paper by Coronado and Talmadge[8].

\section{Measurements of Plasma Flows, Rotation Damping, and Radial Conductivity}

Poloidal flows have been measured using Langmuir probe techniques in bias-probe induced flows in IMS as a function of neutral pressure and radial position. By disconnecting the bias midway through the discharge, measurements of the decay rate of the flow have also been obtained for comparison to damping rates predicted by the neoclassical theory just discussed. By variation of bias electrode position, applied voltage and neutral pressure, radial electrical conductivities have been calculaied for comparison to theory. 
To measure the poloidal flow, a paddle/double probe assembly is oriented such that the paddles face in the positive and negative poloidal directions and are biased into ion saturation. The difference in signals to the two faces of the probe contains the directed ion flow associated with the bulk plasma rotation. The probe direction is alternated between discharges (at the same location) to 'balance' out differences in signals due to external circuitry or effective probe area.

The double probe assembly on the probe stalk also gives data on the floating potential. More detailed experiments measuring the plasma electric field response using emissive probes will be discussed below. By using ion saturation current and floating potential data, profiles of flow velocity based on the ion pressure gradient and the $\mathrm{E} \times \mathrm{B}$ velocity can be calculated for comparison to the measured velocity. The radial profiles resulting from these measurements and calculations are shown in Figure 3 and 4 for two bias-probe locations and an applied potential of $120 \mathrm{~V}$. The profiles show reasonable agreement between the measured flow and that calculated from the floating potential and ion-saturation profiles with the exception of an $\sim 5$ $\mathrm{mm}$ region around the bias electrode location. This agreement indicates that the flow is a result of the radial electric field set up by the biased electrode.

Measurements of the poloidal flow decay have been made by removing the bias from the electrode midway through the discharge. The evolution of the rotation is measured with a $5 \mathrm{MHz}$ sampling and fit to an exponential decay. The measurements have been made for several neutral fill pressures at two radial locations in an attempt to observe the effects of both neutral collision rates and viscosity on the poloidal damping. The results are plotted in Figures 5 and 6 along with the damping rates predicted from neoclassical theory for the experimental conditions. These comparisons show that the linear proportionality of the damping rate to the neutral pressure predicted by the theory is observed in the experimental data but with some deviations at the higher pressures. In general, however, the theory tends to underestimate the experimental results by up to a factor of two. At present, the viscosity effect is calculated using a model which is known to underestimate this contribution. Theoretical work is in progress to improve this estimation.

The plasma potential profile has also been measured under a range of biasing locations, voltages and pressures using emissive probe techniques. The radial electric field is inferred from the potential profile and the radial electrical conductivity is calculated from the electric field and the measured current to the biasing electrode. For purposes of this calculation, the return radial plasma current density required to balance the electrode current from quasi-neutrality is assumed to be uniform into the biased magnetic surface. Figures 7 and 8 show how the potential profiles, and therefore radial electric fields, can be varied by changing the position of the biasing electrode and the applied bias, respectively. Figure 9 shows how changing the neutral gas fill pressure effects the radial potential profiles. The profiles are qualitatively consistent with the model in that the bias current does not change significantly between the three cases, but the electric field is clearly seen to be reduced at higher fill pressures, implying an increase in the radial conductivity as predicted. Preliminary calculations of the radial conductivity agree well with the model in the periphery of the plasma, but deviate significantly near the axis. 
Several refinements to these preliminary measurements are planned for the upcoming experimental run period. Measurement errors in rotation decay will be reduced by modulating the bias potential resulting in the ability to examine muitiple decays within a single, reasonably steady, discharge. Signal averaiging techniques can then be employed to remove errors associated with fluctuation effects in a single decay. More importantly, efforts will be strengthened to look at the important component of viscosity to the rotation damping. The present experiments are dominated by the effects on neutrals on the damping by the need to run at pressures $\geq 20 \mu$ Torr (to achieve breakdown). Additionally, viscosity plays a stronger role where the ripple is largest, in the present IMS configuration near the plasma edge where fluctuations are largest as well. The relative effects of the viscosity on the damping will be increased by using a filament for preionization to lower the operational limits on neutral fill pressure, and by using a vertical field to shift the magnetic surfaces which significantly increases the ripple (and therefore viscous effects) in toward the axis. Improved estimates of the theoretical viscosity contribution will be determined through either application of a code to get the Hamada coordinates for the particular configuration, or through a fully numerical technique.

\section{Direct Orbit Loss and Plasma Potential Structures}

Theories of particle containment in toroidal systems are based upon the quiding-center drift approximation. No experimental work has been done on when the drift approximation is justified. Of particular importance is the question of the effects of orbit size in relation to magnetic field gradient scale-length, a question currently under investigation by Boozer and Yao.

An ion gun has been ciesigned to enable the launching of ions with energies between 10 and $100 \mathrm{eV}$ at variable pitch with respect to the magnetic field. Using a Li6 source, and with the ability to vary the magnetic field strength in IMS, ion gyro-radii can be varied between 2 and $20 \mathrm{~mm}$ and launched with a pitch near 90 degrees. Monitoring the lost ions on the divertor strike plates as a function of launch parameters and magnetic field topology will give data for comparison to guiding center codes on expected loss locations.

Direct loss orbits in stellarators can also drive potential variations on magnetic surfaces and radial electric fields. The perpendicular currents associated with this loss could impart a J $\times \mathrm{B}$ torque to the plasma as well. Little work has been done to date, however, on the effects of electron-cyclotron resonance heating on poloidal potentials and radial electric fields, and the resulting transport, especially in stellarators. IMS would offer a unique opportunity for such investigations through instrumentation of the many divertor areas for determination of the location and energy of lost particles. Variations in the direct loss rate can be achieved by alteration of the helical ripple profile, variation of the resonance location, and through increased ECRH power. Measurements of asymmetric loss flows will be correlated with plasma potential profiles under varying experimental conditions. 


\section{New Concepts}

The similarities of confinement scaling in tokamaks and stellarators has motivated investigations into possible configurations which could provide needed insight into this common behavior. Quasi-helically symmetric (QHS) stellarators, because of the presence of a high degree of helical symmetry in a toroidal device, can be considered a 'currentless tokamak', with similarly excellent single-particle confinement. Because this concept bridges the gap between asymmetric currentless stellarators and symmetric current-driven tokamaks, it would provide a unique opportunity to explore fundamental physics questions in toroidal confinement. The QHS configuration actually has reduced ion banana widths and neoclassical transport predicted as compared to an equivalent tokamak.

To examine the potential role of QHS configurations in the toroidal confinement program, various QHS magnetic structures based on cases discovered at Garching by Nührenberg were investigated. These cases were modified to suit goals of magnetic well, near helical symmetry and reduced aspect ratio. A configuration was found which had a magnetic well to near the plasma edge, very low non-symmetric components at low betas, toroidal curvature term in $|\mathrm{B}|$ equivalent to a conventional stellarator at aspect ratio 240, reduced Pfirsch-Schlüter currents, and nzoclassical transport over 2 orders of magnitude below a conventional stellarator. Neoclassical transport was also factor of 2-3 below an equivalent tokamak, and with a similar possible reduction of 3 in anomalous transport as well, based on Lackner scaling. These investigations led to the development of the concept of the HSX device, which has been separately submitted for evaluation.

\section{REFERENCES}

[1] DIAMOND, P.H., KIM, Y.B, Phys. Fluids B 3 (1991) 1626.

[2] SHAING,K.C., CRUME JR.,E.C., AND HOULBERG,W.A., Phys. Fluids B2, (1990) 1492.

[3] GROEBNER,R.J., BURRELL, K.H., AND SERAYDARIAN, R.P., Phys. Rev. Lett., 64 (1990) 3015.

[4] TAYLOR, R.J., BROWN, M.L., FRIED, B.D., GROTE, H., LIBERATE, J.R., MORALES, G.J., AND PRIBYL, P., Phys. Rev. Lett. 63, (1989) 2365.

[5] WEYNANTS, R.R., AND TAYLOR, R.J., Nucl. Fusion, 30 (1990) 945.

[6] ITOH,S.I. AND ITOF_, K., Nucl. Fusion, 29 (1989) 1031.

[7] ROZHANSKII, V.A., AND TENDLER, M.B., JETP Lett. $\underline{53}$ (1991) 82.

[8] CORONADO, M., AND TALMADGE, J.N., TSL Report 11-92-1; also, submitted for publication to Phys. Fluids. B 


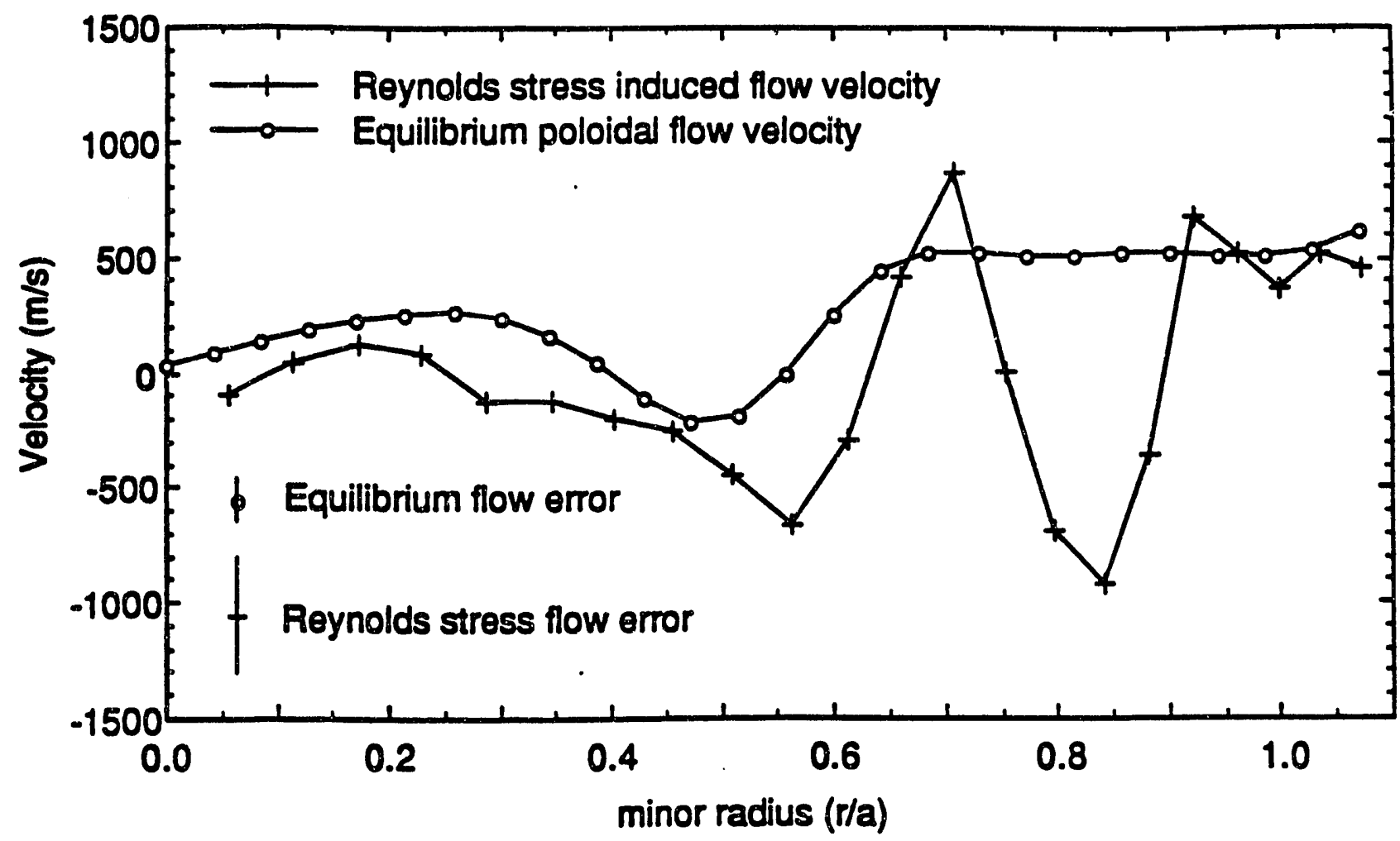

FIG. 1. Comparison of Reynolds stress driven poloidal flow, and the flow computed from the electric field, density, and ion pressure profiles. 


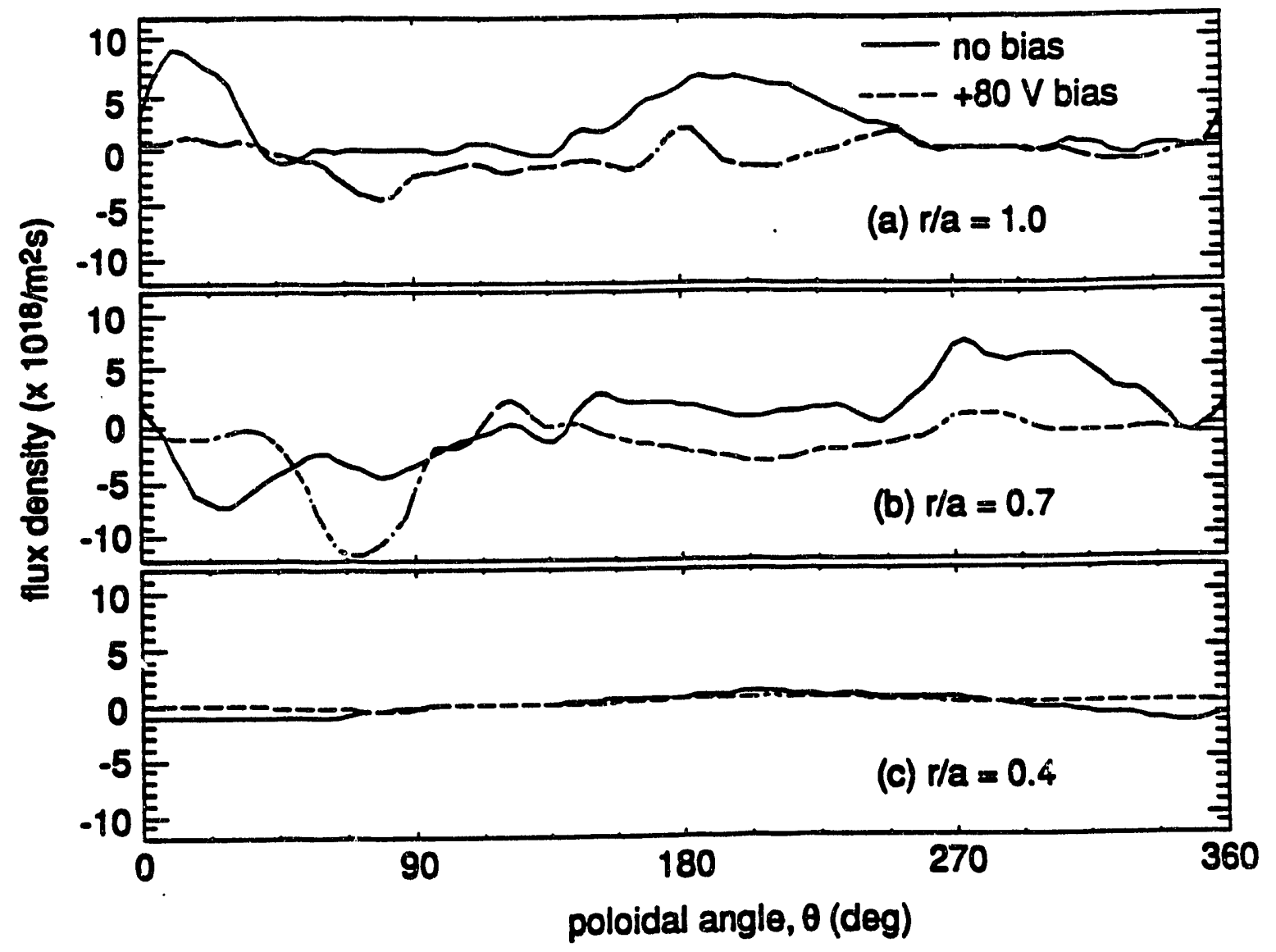

FIG. 2. Fluctuation-induced particle transport in IMS for biased and unbiased conditions, decomposed onto a magnetic flux surface for three minor radii: (a) r/a $=1.0 ;(b)$ ria $=$ $0.7 ;$ (c) $r / a=0.4$. 


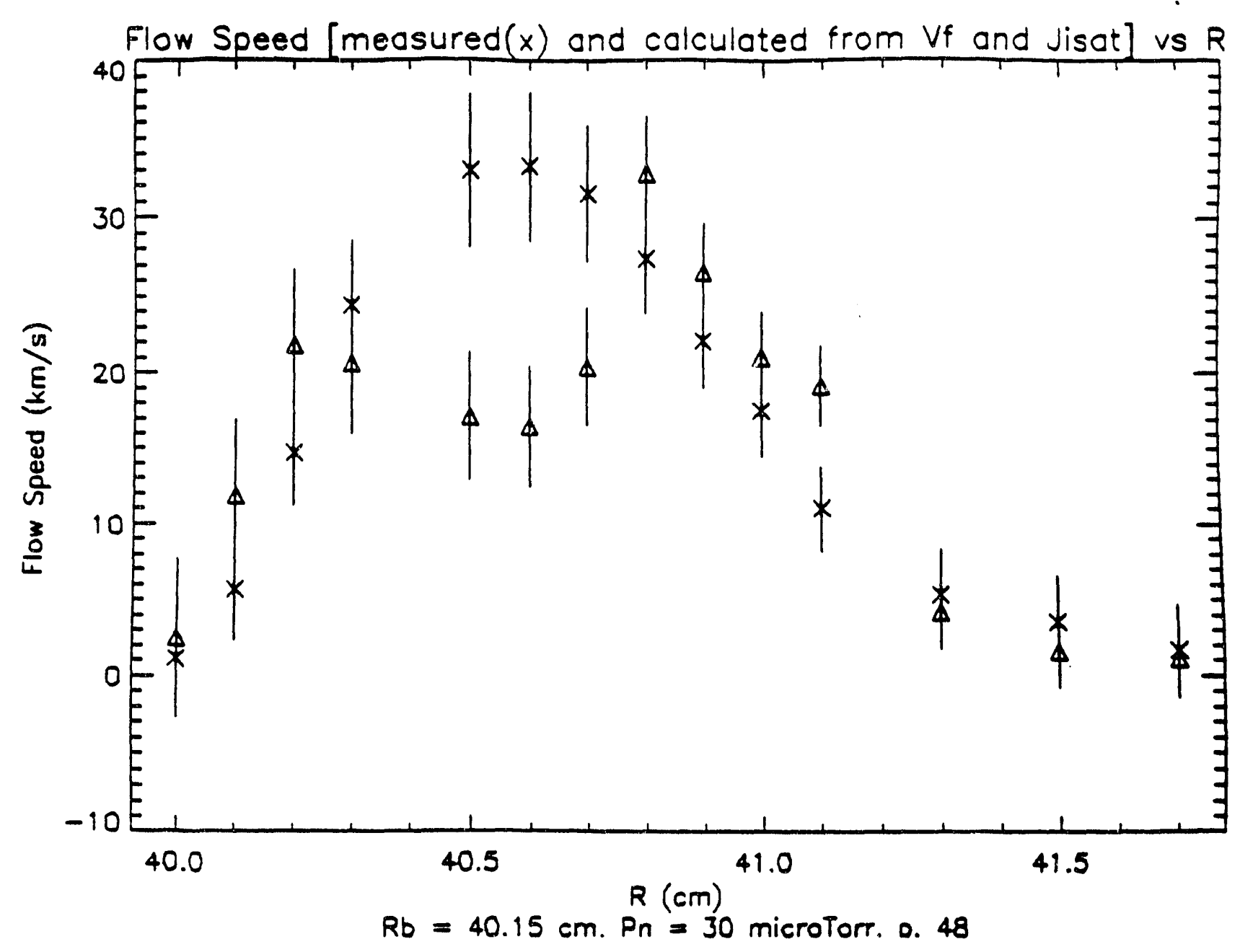

Figure 3

Poloidal Flow Speed vs. Major Radius

$x$ - measured, $\Delta-E \times B$ (Calculated from the floating potential and Ion saturation current measurements)

For $R$ (bias) $=40.15 \mathrm{~cm}, \mathrm{~V}$ (bias) $=120 \mathrm{~V}$, Neutral Pressure $=30 \mu$ Torr 


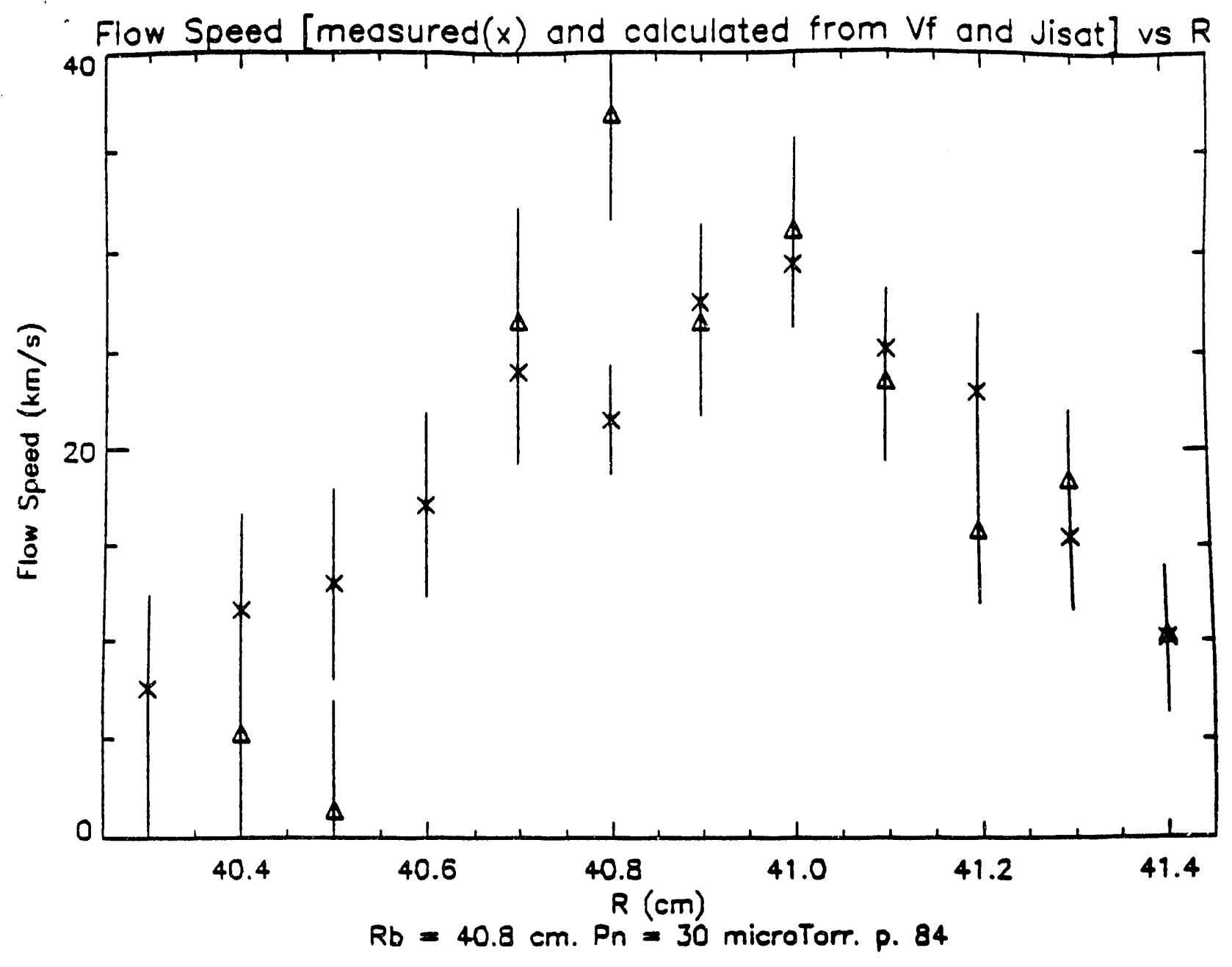

Figure 4

Poloidal Flow Speed vs. Major Radius

$x$ - measured, $\Delta$ - E X B (Calculated from the floating potential and Ion saturation current measurements)

For $R$ (bias) $=40.8 \mathrm{~cm}, V$ (bias) $=120 \mathrm{~V}$,

Neutral Pressure $=30 \mu$ Torr 


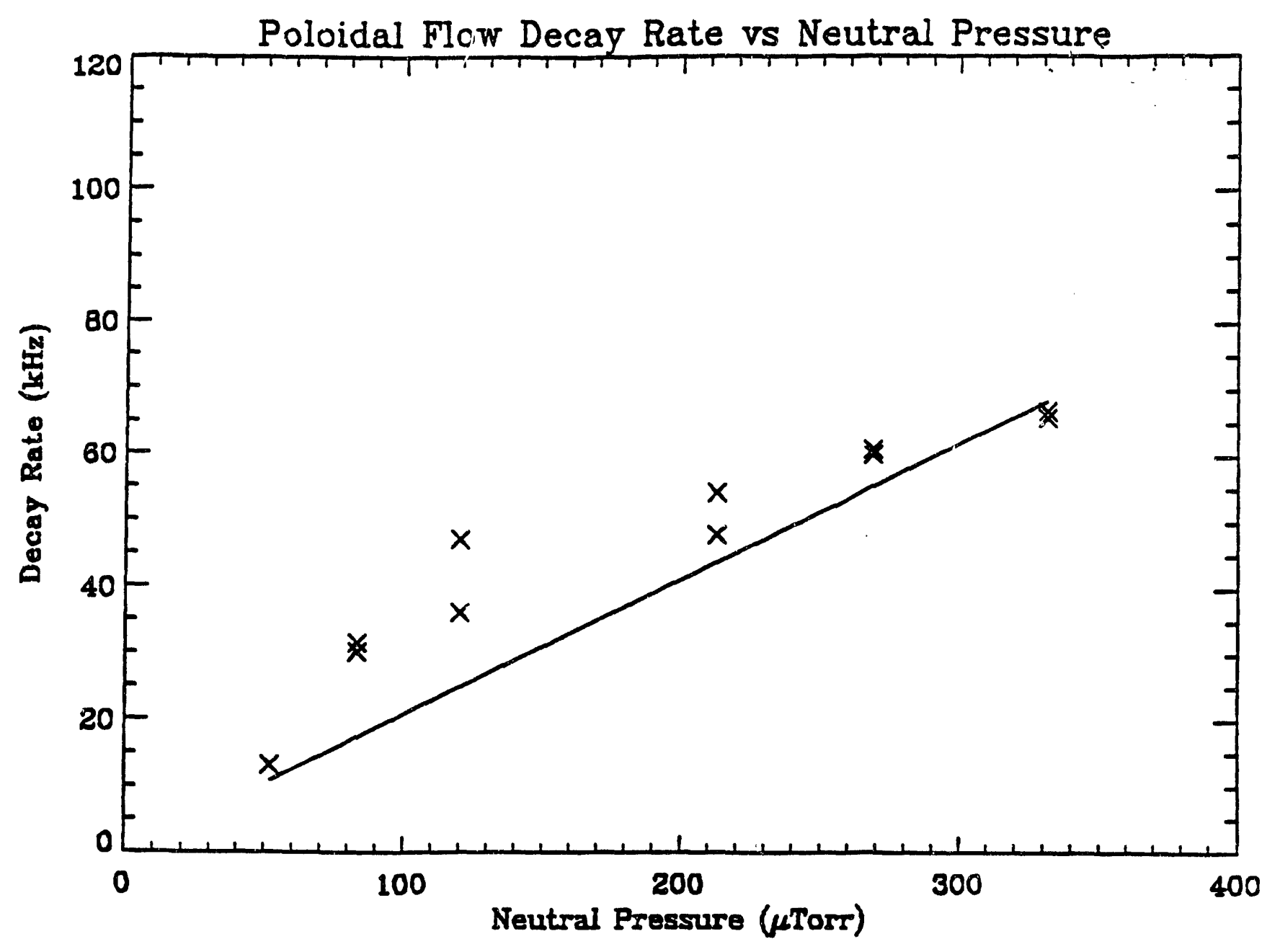

Figure 5

Poloidal Flow Decay Rate vs. Neutral Pressure at $R=40.8 \mathrm{~cm}$ $x$ - measured, line - neoclassical theory For $R$ (bias) $=40.15 \mathrm{~cm}, \mathrm{~V}$ (bias) $=120 \mathrm{~V}$ 


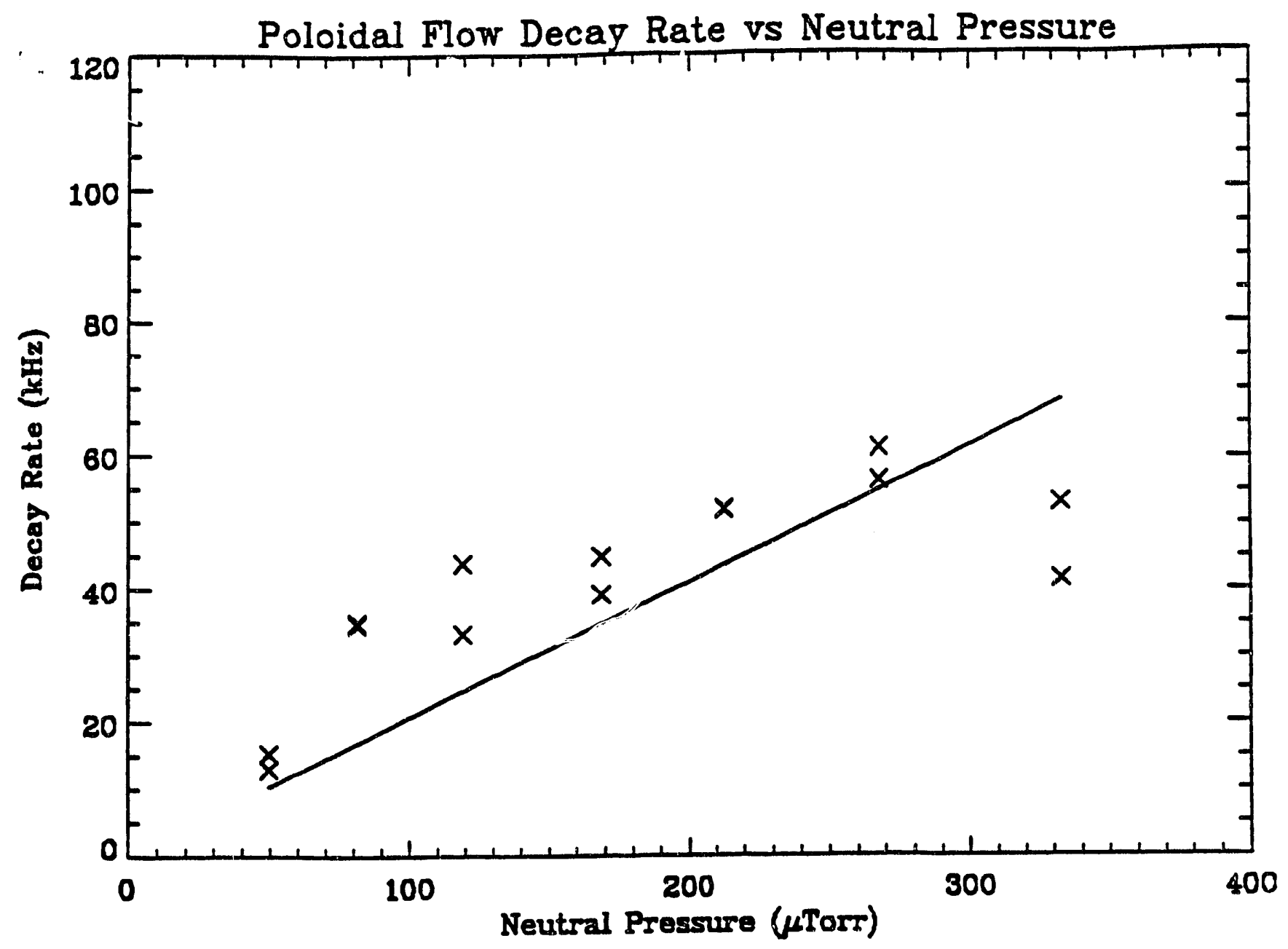

Figure 6

Poloidal Flow Decay Rate vs. Neutral Pressure at $R=41.0 \mathrm{~cm}$ $x$ - measured, line - neoclassical theory

For $R($ bias $)=40.8 \mathrm{~cm}, \mathrm{~V}$ (bias) $=120 \mathrm{~V}$ 


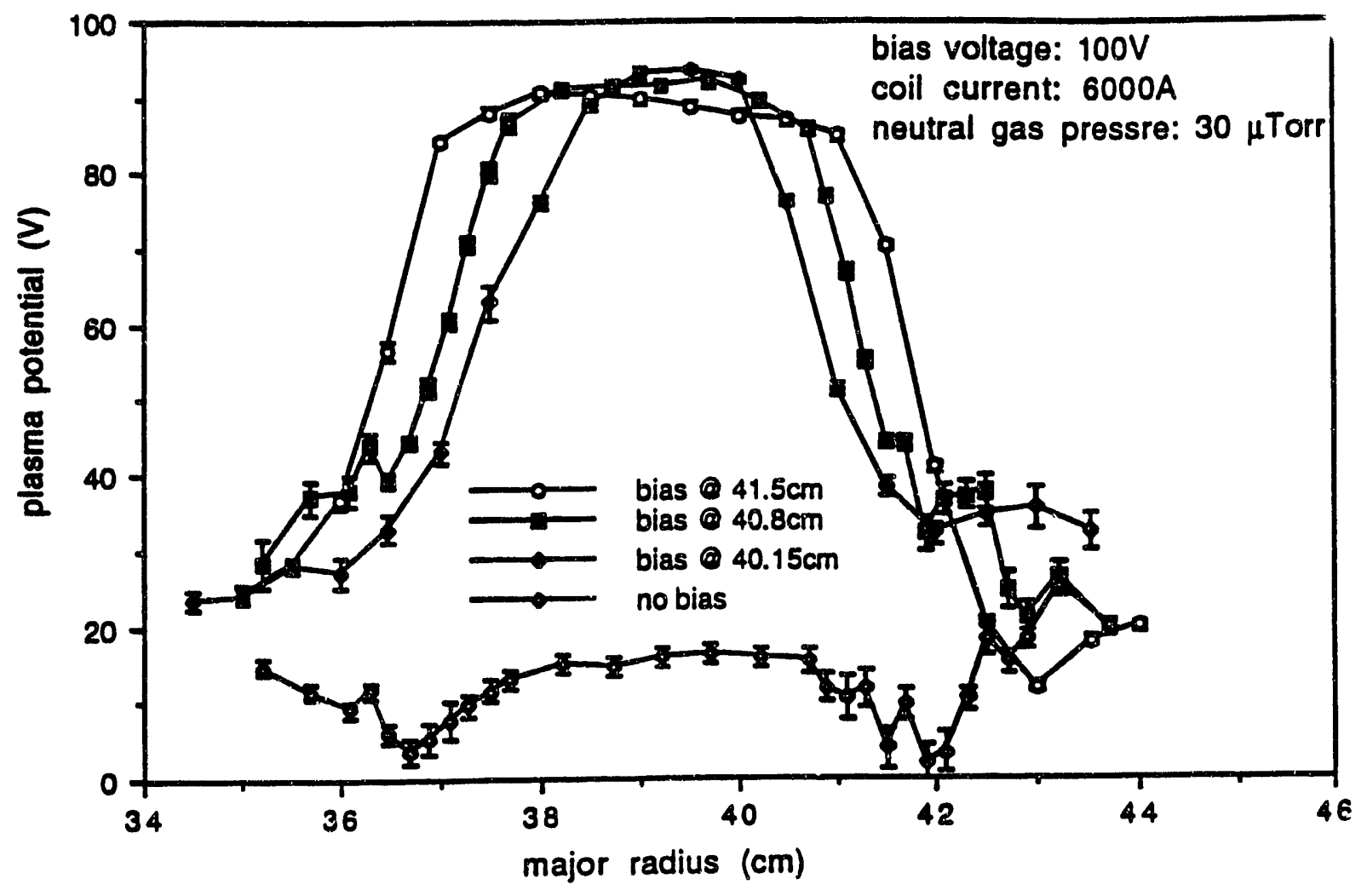

Fig. 7 effects of bias location on plasma potential 


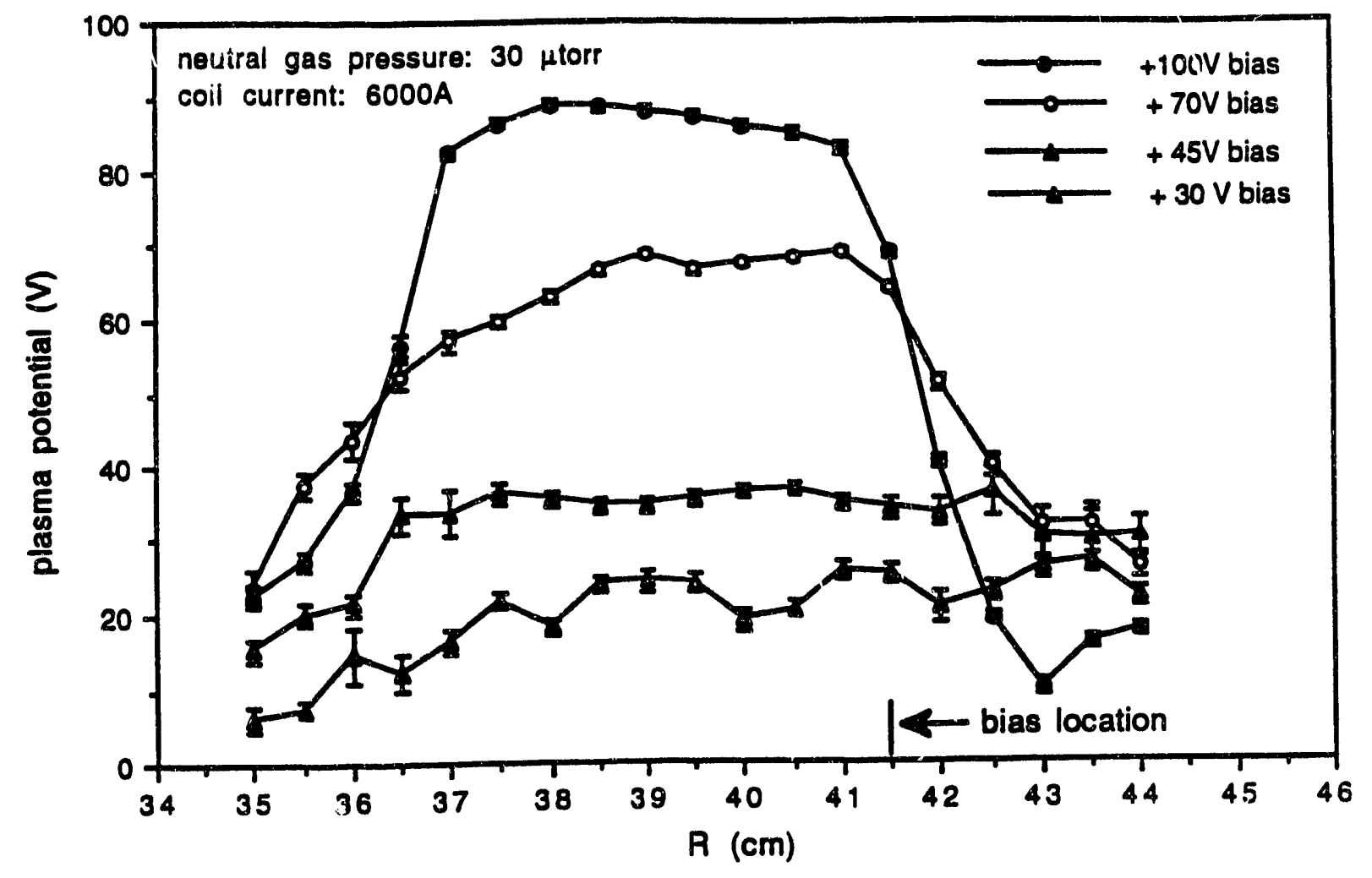

Fig. 8 effects of bias voltage of plasma potential

\section{DISCLAIMER}

This report was prepared as an account of work sponsored by an agency of the United States Government. Neither the United States Government nor any agency thereof, nor any of their Government. Neither the United States Gor implied, or assumes any legal liability or responsibility for the accuracy, completeness, or usefulness of any information, apparatus, product, or process disclosed, or represents that its use would not infringe privately owned rights. Reference herein to any specific commercial product, process, or service by trade name, trademark, manufacturer, or otherwise does not necessarily constitute or imply its endorsement, recommendation, or favoring by the United States Government or any agency theieof. The vicws and opinions of authors expressed herein do not necessarily state or reflect those of the United States Government or any agency thereof. 


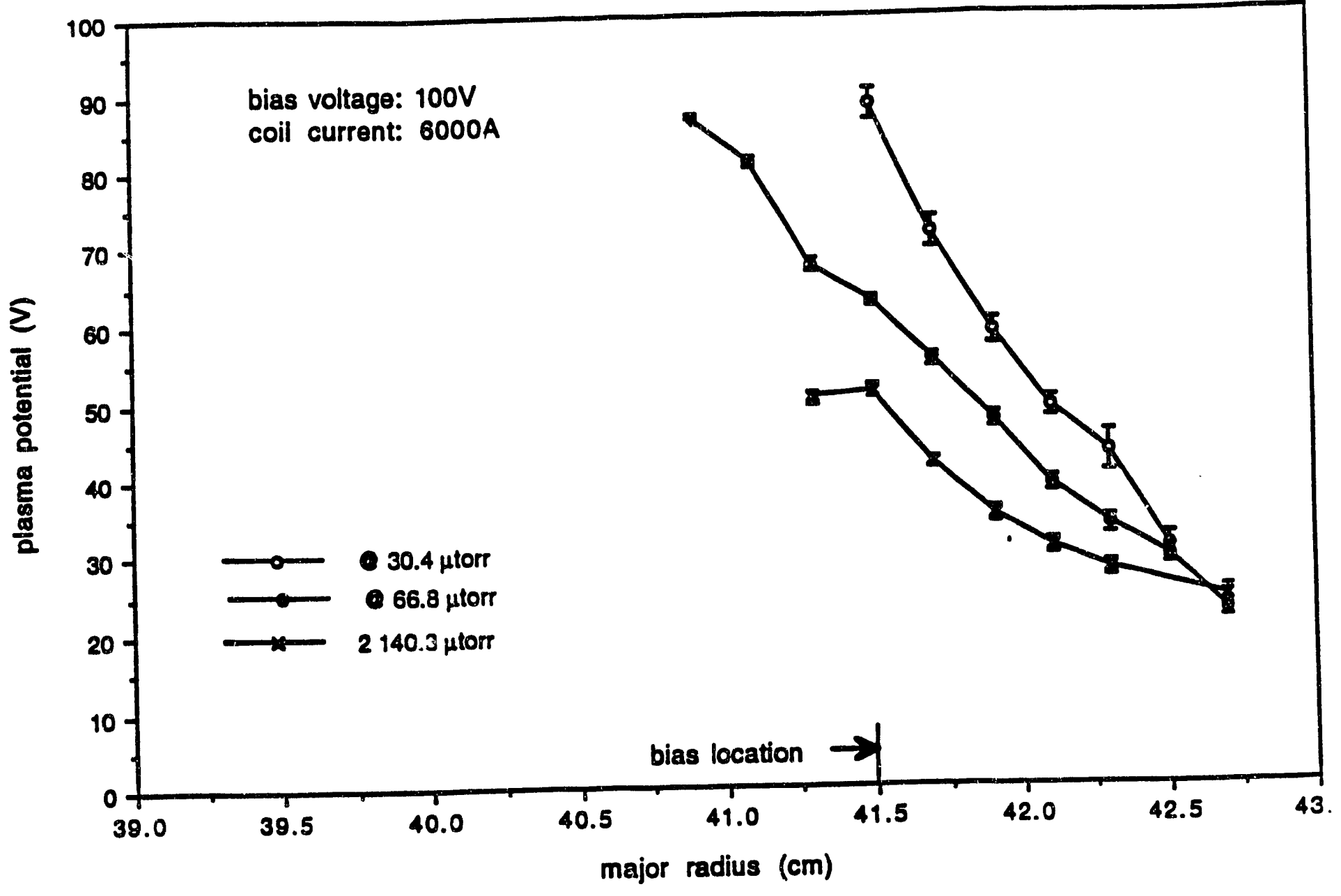

Fig 9 Plasma potential radial-profile variation with neutral gas pressure. 
DATE FILMED 218193 


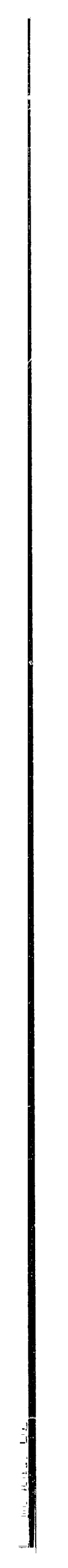

